\title{
Operadores de aproximación en el decir y de atenuación en griego antiguo: la partícula $\pi 0 v^{*}$
}

\author{
José Miguel Jiménez Delgado \\ Universidad de Sevilla \\ jmjimdelg@us.es
}

ORCID iD: https://orcid.org/0000-0003-2510-2786

\section{Approximators and hedges in Ancient Greek: the particle $\pi$ ov}

\begin{abstract}
Este trabajo constituye un intento de explicación de los valores tradicionalmente considerados modales de la partícula $\pi$ ov. De acuerdo con nuestro análisis, $\pi$ ov no se usa como una partícula de modalidad epistémica, sino que esos valores son más propios de un operador de aproximación en el decir y de atenuación. Esta explicación casa mejor con los demás usos reconocidos para la partícula y, como veremos, permite entender los supuestos usos modales de forma más consistente.
\end{abstract}

Palabras clave: Adverbios de lugar indeterminado; Partículas modales; Operadores; Aproximantes; Atenuantes; (Inter)subjetificación.
This paper offers an alternative explanation for the traditional modal uses of the Greek particle $\pi$ ov. According to our analysis, $\pi$ ov is never employed as a modal particle but as an approximator and as a hedge. This explanation is more consistent with other uses and enables a better understanding of the so-called modal ones.

Key words: Indefinite local adverbs; Modal Particles; Sentence adverbs; Approximators; Hedges; (Inter)subjectification.

Cómo citar este artículo / Citation: Jiménez Delgado, José Miguel 2019: «Operadores de aproximación

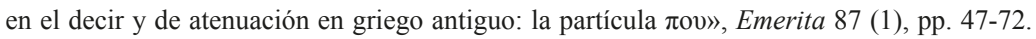

* Este trabajo ha sido realizado en el marco del proyecto de investigación FFI201565541-C3-1-P, financiado por la DGICYT del Ministerio de Economía, Industria y Competitividad. El autor quisiera hacer constar su agradecimiento a Luz Conti, así como a los dos informantes anónimos.

Copyright: (C) 2019 CSIC. Este es un artículo de acceso abierto distribuido bajo los términos de la licencia de uso y distribución Creative Commons Reconocimiento 4.0 Internacional (CC BY 4.0). 


\section{Introducción}

El presente trabajo constituye un intento de analizar la partícula griega $\pi$ ov desde una perspectiva novedosa. En este sentido, se analizan los usos tradicionalmente considerados modales de la partícula, que originalmente significa 'en algún lugar, en un sitio cualquiera', desde la perspectiva de los operadores de aproximación en el decir y de la de los operadores de atenuación o cortesía. Este tipo de operadores se desarrolla, con frecuencia, a partir de elementos caracterizados por la indeterminación, como se ha observado en estudios aplicados a lenguas europeas modernas.

El artículo tiene la siguiente estructura: en primer lugar, se hará un breve repaso de los principales valores de la partícula, para abordar, después, las interpretaciones que se han dado a su supuesto uso modal. En la tercera sección, se explican las características que definen a los operadores de aproximación en el decir y de atenuación o cortesía. Como término de comparación, se toman las locuciones españolas «de alguna manera» y sus variantes «de alguna forma» $\mathrm{y}$ «de algún modo». En la cuarta sección, se propone interpretar $\pi$ ou modal como aproximante y atenuante (denominaciones alternativas a operador de aproximación en el decir y de atenuación o cortesía, respectivamente) en consonancia con otros usos no literales y, en la quinta, se explica la evolución de adverbio de lugar indeterminado a operador de aproximación en el decir y de atenuación, con ejemplos de esos valores. Finalmente, se ofrecen las conclusiones pertinentes.

\section{La particula nov como adverbio de lugar indeterminado y usos no literales}

La partícula $\pi$ ov es un derivado del tema indefinido-interrogativo indoeuropeo * $k^{u} o / e h_{2}$ - con una terminación de genitivo temática. En griego antiguo, la forma interrogativa $\pi$ oṽ se diferenciaba de la indefinida por el acento. Ambas formas tienen un significado original de tipo local, como es habitual en jónico-ático para esa terminación de genitivo en formas adverbiales (la forma eolia equivalente a $\pi$ ov es $\pi$ or, cf. Wackernagel 1895). En resumidas cuentas,

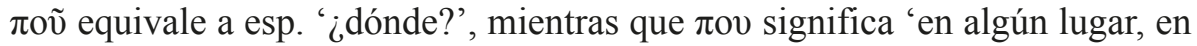
alguna parte'. A partir de ese significado los diccionarios (LSJ; Bailly 2000; Montanari 2015) reconocen una serie de valores no literales que se pueden agrupar de la siguiente forma: 
1. Como adverbio de aproximación o aproximativo, generalmente limitando cantidades expresadas mediante numerales y cuyo valor exacto se desconoce:

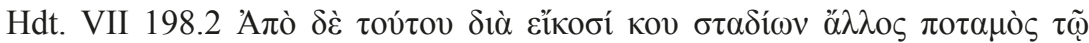

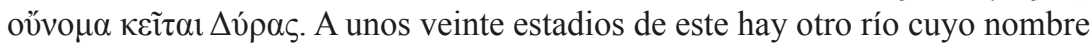
es Diras ${ }^{1}$.

Este uso es especialmente frecuente en las Historias de Heródoto, pero también se documenta en otros autores:

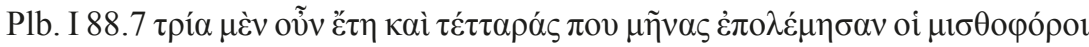

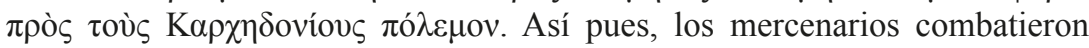
contra los cartagineses tres años y unos cuatro meses.

2. Como adverbio de probabilidad o posibilidad, asociado, por ejemplo,

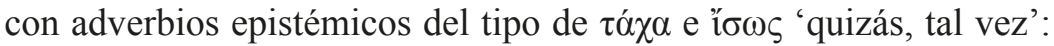

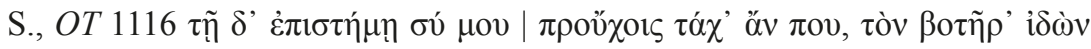
rápos. Es posible que tú me aventajes en conocimiento, si viste al pastor hace un tiempo.

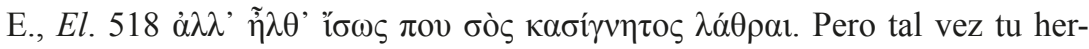
mano ha venido en secreto.

A pesar de que los léxicos suelen subrayar esta asociación, que resulta un tanto redundante, es poco frecuente ${ }^{2}$.

3. Como refuerzo de otros elementos indefinidos:

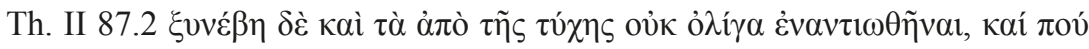

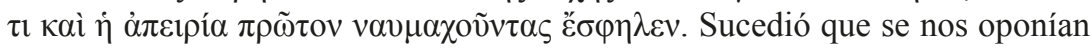
no pocas circunstancias que dependen del azar y, hasta cierto punto, también la inexperiencia nos jugó una mala pasada en la que fue nuestra primera batalla naval. (Asociado con $\tau$, pronombre indefinido)

${ }^{1}$ Los textos griegos son los de las ediciones recogidas en el $T L G$; las traducciones son propias y tratan de reflejar los valores de la partícula objeto de estudio.

${ }^{2} \mathrm{La}$ asociación de la partícula con î $\sigma \omega \varsigma$ es rara y en griego clásico, aparte del ejemplo euripideo, hay otro platónico, Pl., Ap. 39b. La asociación con $\tau$ ó $\chi \alpha$ tampoco es frecuente y se documenta también en Pl., Phdr. 256c. 


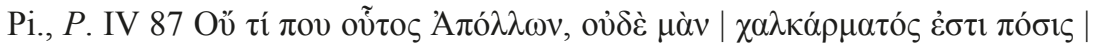

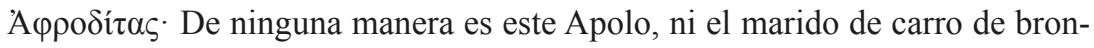
ce de Afrodita. (Oú $\tau i ́$ đov es una combinación relativamente frecuente)

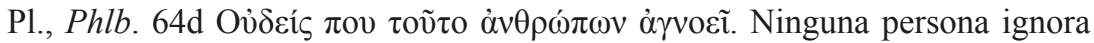

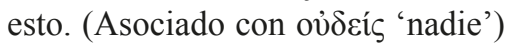

3. La partícula $\pi 0 v$ como operador modal de carácter epistémico: ¿certeza, probabilidad o posibilidad?

Como acabamos de ver en la sección anterior, tradicionalmente se ha considerado que la partícula $\pi$ ov, además de como adverbio de lugar y como adverbio de aproximación, es una partícula de carácter epistémico que aporta un matiz de probabilidad o posibilidad a su oración difícil de traducir. Con respecto a las interpretaciones más frecuentes, Koier 2013, pp. 166-172, 299308 recoge las siguientes:

1. Partícula epistémica de probabilidad o posibilidad (Denniston 1954, pp. 490-495; Wakker 1994, p. 362; Sicking 1993, pp. 57-61; Wackernagel 1895, pp. 21-25; Bolling 1929; Schwyzer-Debrunner 1950, p. 579).

2. Partícula epistémica de certeza (Wackernagel 1895, pp. 21-25).

3. Partícula irónica, cuando se pretende marcar la proposición como posible o probable, a pesar de que el hablante está plenamente seguro de su veracidad (Denniston 1954, pp. 490-495; Bodin-Mazon 1912, pp. 358-359).

4. Partícula interpersonal del tipo de los atenuantes (Sicking 1993, pp. 5761).

5. Marcador del contenido de la proposición como información fácilmente accesible por su obviedad o trivialidad (Sicking 1993, pp. 57-61; Bodin-Mazon 1912, pp. 358-359).

6. Aproximante con el que se aporta vaguedad o inespecificación a lo dicho (Schwyzer-Debrunner 1950, p. 157, n. 3; Sicking 1993, pp. 57-61; Slater 1969 s. u.; Italie 1955 s. u.; Scolnicov 2003, pp. 34, 40 y 81).

Debe tenerse en cuenta que, en general, todas las interpretaciones giran en torno al valor epistémico de probabilidad o posibilidad. En este sentido se puede entender la descripción de sus valores modales que ofrece Wackernagel 1895, p. 22: «Schon bei Homer dient $\pi$ ov bekanntlich nicht bloss im 
lokalen sinne, sondern auch und noch häufiger im sinne von 'gewiss' 'doch wohl' in behauptungen, von deren richtigkeit man überzeugt ist, die man aber nicht beweisen kann». Más interesantes son las consideraciones que realiza Sicking 1993, p. 59 sobre el valor modal de $\pi$ ov, a partir de un sentido básico que sería el siguiente: "with $\pi$ ov a speaker presents his statement as a surmise whose accuracy he does not vouch for so that disputing it need not impair the basis for an understanding between the two partners in a conversation». Partiendo de ese valor básico, Sicking observa que la partícula se puede usar como atenuante o aproximante, por ejemplo, para evitar la pedantería cuando se introducen proposiciones obvias, para facilitar la conversación o para aminorar la fuerza argumentativa de determinadas afirmaciones. Obsérvese que el valor básico atribuido a tov modal entra dentro de la consideración de que expresa probabilidad o posibilidad, aunque se trata de un valor muy matizado, ya que con esta partícula no se pondría en cuestión la realidad o factualidad de su oración como sí se hace cuando se usan los adverbios epistémicos propiamente dichos.

Sea como fuere, la visión tradicional, en virtud de la cual $\pi$ ov expresa probabilidad o posibilidad, encontraría cierto apoyo en las escasas consideraciones que nos han llegado de los gramáticos antiguos sobre el valor de la partícula. Así, son varios los escolios platónicos en los que se glosa $\tilde{\eta} \pi \mathrm{ov}$ como equivalente de ǐ $\sigma \omega \zeta$, $\sigma \chi \varepsilon \delta o ́ v$ 'quizás, tal vez’3. Esta equivalencia aparece reflejada en algunos léxicos bizantinos, como la Suda: $\tilde{\eta} \pi 0 v$, ไ̌ $\sigma \omega \varsigma, \sigma \chi \varepsilon-$

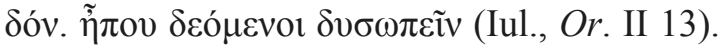

Sin embargo, en el Etymologicum Gudianum la equivalencia se da con adverbios evidenciales del tipo de őv $\tau \omega \varsigma$ 'realmente' o $\alpha \lambda \eta \theta \tilde{\omega} \varsigma$ 'verdade-

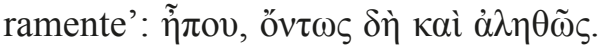

Ambas interpretaciones quedan recogidas en el Etymologicum Magnum, que aporta otras consideraciones que merece la pena reseñar:

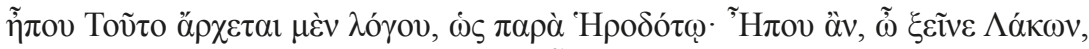

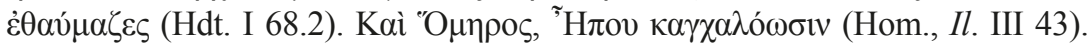

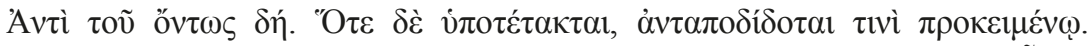

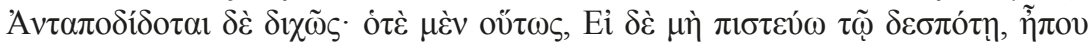

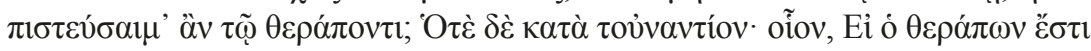

${ }^{3}$ Véanse los escolios a Euthphr. 4a, Plt. 285d, Amat. 132b, R. 450d, 595c, Ep. 360b. En la tradición manuscrita de estos escolios hay cierta confusión de $\tilde{\eta} \pi \mathrm{ov}$ con $\ddot{\eta} \pi \mathrm{ov}$. 


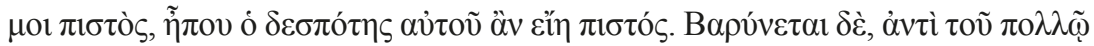

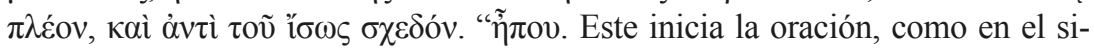
guiente pasaje de Heródoto: «Sin duda, forastero laconio, te maravillarías»; véase también en Homero: «Sin duda, se reirán». Equivale a őv $\tau \omega \varsigma \delta \eta ́$ 'realmente'. Cuando se pospone, se corresponde con algo colocado delante. Se corresponde de dos maneras: unas veces así «Si no confiara en el amo, ciertamente podría confiar en el sirviente», otras al contrario «Si el sirviente es digno de mi confianza, ciertamente su amo puede serlo también». Tiene acento grave con el

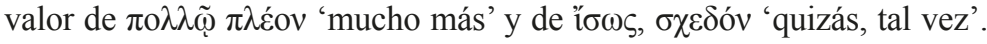

Se debe tener en cuenta, en primer lugar, que hay cierta confusión entre $\tilde{\eta}$ y los escolios platónicos se establece con ク̋ $\pi 00 \gamma \varepsilon^{4}$. La impresión es que se está combinando, por un lado, el valor de $\tilde{\eta}$, partícula afirmativa ${ }^{5}$ equivalente a

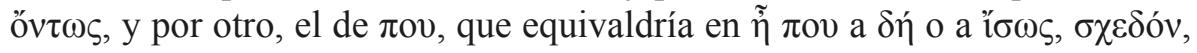

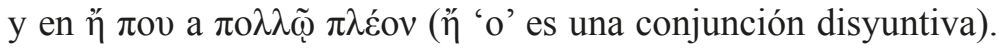

Con todo, es dudoso que la identificación con ǐ $\sigma \omega \varsigma, \sigma \chi \varepsilon \delta \delta$ óv tenga que ver con el uso epistémico de estos adverbios, cf. n. 14. En este sentido, Apolonio Díscolo considera que $\pi \mathrm{ov}$, junto con $\theta \eta v, \rho \alpha$ y $v$, equivale a $\delta \eta ́$, si bien no se trata de un conector, sino de un adverbio:

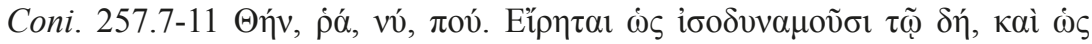

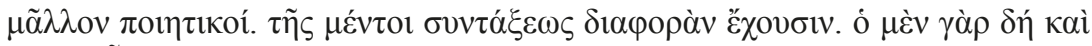

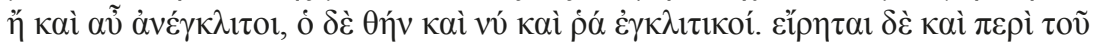

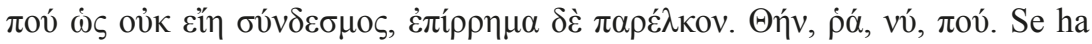
dicho que equivalen a $\delta \eta ́$ y que son más poéticos. Sin embargo, tienen una diferencia de construcción, pues $\delta \eta ́$, , y $\alpha \tilde{u}$ no son enclíticos, mientras que

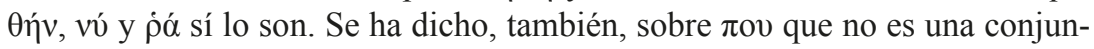
ción, sino un adverbio redundante.

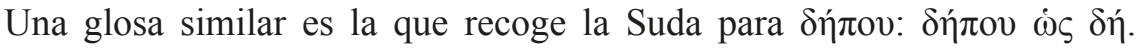
«

Es difícil interpretar esta equivalencia, pero llama la atención que la Suda considere que $\pi$ ov es un añadido «expletivo» a la partícula $\delta \eta ́$ al igual que Apo-

${ }^{4}$ Escolios a Plt. 285d, Amat. 132b, R. 450d, 595c, Ep. 360b.

${ }^{5}$ Cf. Denniston 1954, pp. 279-282. Se trata de un adverbio epistémico que subraya la certeza de su proposición. 
lonio lo califica de «adverbio redundante». Véase también la glosa de $\tilde{\eta} \pi$ Tov en

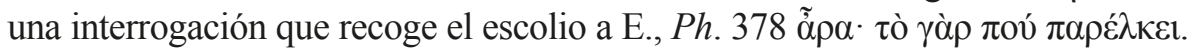

Más allá de estas glosas y de sus dificultades de interpretación, Koier 2013 ha puesto en cuestión la visión tradicional de esta partícula sobre la base de un estudio contrastivo apoyado en presupuestos procedentes de los estudios acerca de elementos similares en lenguas modernas. Koier compara el uso de $\pi$ ov con el de una partícula holandesa similar, ergens 'en alguna parte', que también tiene un valor local indefinido original y valores no literales que derivan de este ${ }^{6}$. El análisis de esta partícula se basa en encuestas realizadas por la propia Koier y muestra cómo una partícula local de carácter indefinido puede derivar hacia usos no literales. En el caso de la partícula griega, Koier basa su análisis en un buen número de traducciones inglesas, francesas y alemanas. La autora holandesa encuentra las siguientes, por orden de frecuencia, en el corpus en prosa que maneja de época clásica (Koier 2013, p. 193; véanse también pp. 190, 192, 253, 265):

En inglés: ø, I suppose, I think, you know, I presume, surely, may, no doubt, of course, why.

En francés: $\varnothing$, j'imagine, sans doute, je crois, en somme, n'est-ce pas?, peut-on dire, en quelque façon, je pense, peut-être.

En alemán: ø, doch, wohl, doch wohl, ja, doch irgendwie, ja wohl, vermutlich, doch auch, gewiss.

Las traducciones son muy variadas, llamando la atención que, en todos los casos, la opción más seguida es la de dejar la partícula sin traducir, dato que da cuenta de las dificultades que presenta su interpretación. Por lo demás, la partícula no suele verterse con adverbios epistémicos de probabilidad o posibilidad, sino que muchas veces se traduce por expresiones que denotan factualidad. Sobre la base de estas traducciones y sobre una serie de propiedades combinatorias de la partícula, la autora llega a la conclusión de que $\pi$ ov, en sus usos modales, es más bien una partícula con un valor epistémico de certeza que indica que lo que se dice es conocimiento accesible tanto para el hablante como para el oyente. Este conocimiento, o bien pertenece al conocimiento enciclopédico de ambos, o bien es fácilmente deducible de lo dicho.

${ }^{6}$ De acuerdo con el estudio de Koier, ergens también se usa con sentido temporal, como adverbio de aproximación, como refuerzo de elementos indefinidos, para indicar que lo que se expresa se sitúa en el espacio mental de los sentimientos o pensamientos del hablante, o dentro de la esfera de un punto de vista determinado, además de como aproximante. 
De acuerdo con Koier, el argumento más importante para apoyar su análisis es la asociación de esta partícula con proposiciones cuya veracidad no puede ponerse en cuestión (Wackernagel 1895, p. 22; Sicking 1993, p. 59). Es el caso de axiomas como el siguiente:

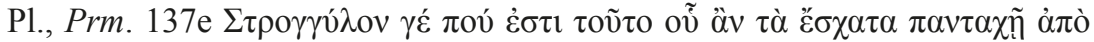

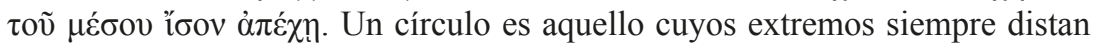
lo mismo del centro.

También es frecuente el uso de la partícula cuando se enuncian hechos atribuidos a los dioses que, en una sociedad como la griega, no se ponen en cuestión:

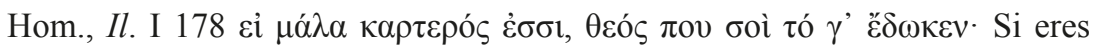
muy fuerte, un dios te lo concedió.

Por otro lado, Koier destaca su frecuente colocación con otros elementos que indican factualidad como la partícula $\delta \eta$, que traduce por 'evidently' siguiendo a van Ophuijsen 1993, pp. 140-146, la partícula afirmativa $\tilde{\eta}$ y el adverbio $\pi \alpha ́ v \tau \omega \varsigma$ con el valor modal de 'certainly'. Especialmente frecuente es la asociación con $\tilde{\eta}$ y, particularmente, con $\delta \eta$, por lo que se suelen escribir los dos elementos juntos ( $\delta \dot{\pi} \pi \mathrm{ov})$ :

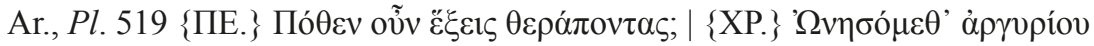
$\delta \eta ́ \pi$ rov. (Pobreza) - Entonces, ¿de dónde vas a sacar a los sirvientes? (Crémilo) Los compraremos con dinero, claro.

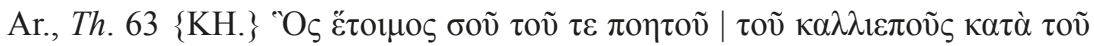

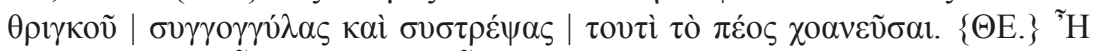

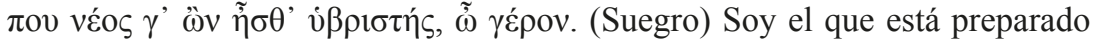
para dar forma en el crisol a esta verga de aquí, redondeándola y condensándola dentro de tu cornisa ${ }^{7}$ y la de tu poeta de hermoso verbo. (Sirviente) Desde luego que eras insolente de joven, anciano.

En ático, especialmente en los diálogos de Platón y, en menor medida, en los diálogos socráticos de Jenofonte, se usan las expresiones $\pi \alpha ́ v \tau \omega \varsigma$ $\delta \eta ́ \pi 0 v$ y $\pi \alpha ́ v \tau \omega \varsigma$ $\pi$ ov como respuesta afirmativa a una interrogación total:

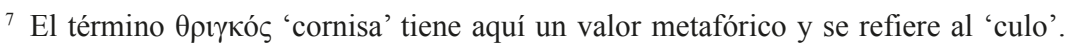




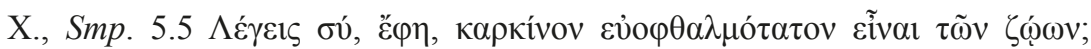

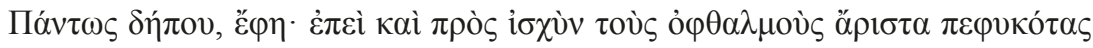

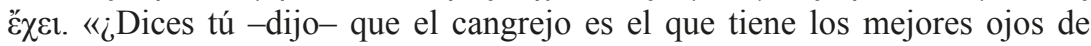
todos los animales? Por supuesto -dijo-, pues tiene los ojos mejor formados por su fuerza».

Otras asociaciones frecuentes de la partícula con valor modal redundarían en esa misma interpretación, especialmente su asociación con verbos de conocimiento, caracterizados por su factividad (Kiparsky-Kiparsky 1970) ${ }^{8}$, y con verbos de lengua en primera persona, con los que el hablante se responsabiliza de la veracidad de lo que dice:

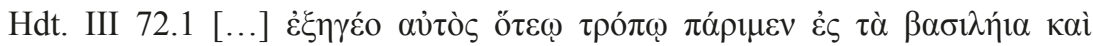

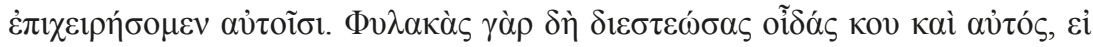

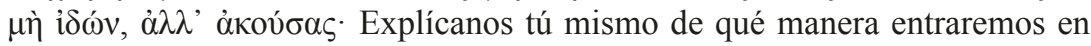
el palacio y atentaremos contra ellos, pues también tú sabes que hay guardias apostados en distintos lugares, si no porque los has visto, al menos porque lo habrás oído.

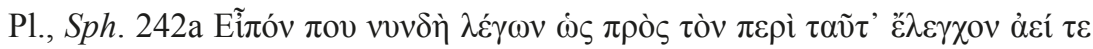

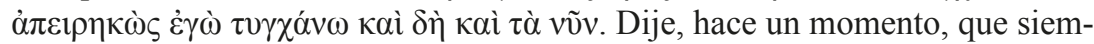
pre me he encontrado sin fuerzas para refutar esto, como también ahora lo estoy.

También es frecuente su asociación con la partícula yó $\rho$, especialmente cuando introduce enunciados explicativos que contienen información accesible tanto para el hablante como para su interlocutor. En este sentido, la

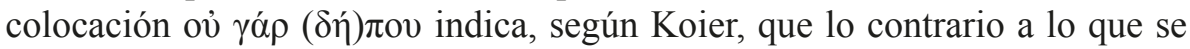
dice sería ilógico o, incluso, ridículo:

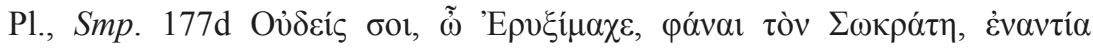

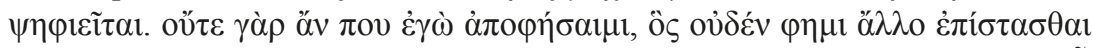

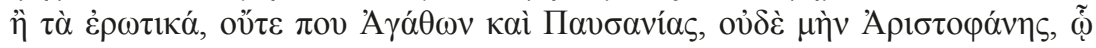

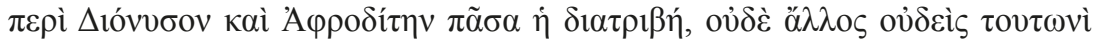

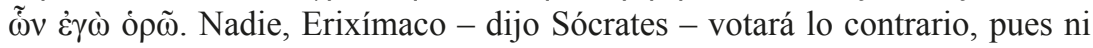

${ }^{8}$ Los predicados factivos presuponen la veracidad de su completiva, mientras que los no factivos dejan esa presuposición sin activar. Sobre la factividad en griego antiguo, véase Huitink 2009 y Rijksbaron 2002, pp. 50-51, 54. 
yo podría negarme, ya que afirmo que no sé nada salvo lo relativo al amor, ni Agatón ni Pausanias, ni tampoco Aristófanes, que pasa todo su tiempo entre Dioniso y Afrodita, ni ningún otro de los que estoy viendo aquí presentes.

Este pasaje constituye parte de la respuesta de Sócrates a la propuesta de Erixímaco de que, una vez terminado el banquete, cada uno realice un discurso en alabanza del Amor. Sócrates responde que la propuesta es muy apropiada, pues es conocido el interés por el Amor tanto por parte de él como del resto de participantes. Recuérdese que Agatón y Pausanias eran amantes.

\section{Operadores de aproximación en el decir y atenuación: de alguna manera, de alguna forma, de algún modo}

En este trabajo se propone interpretar los usos tradicionalmente considerados modales de la partícula $\pi 0 v$ como valores característicos de un operador de aproximación en el decir y de atenuación. Como veremos, la consideración de esta partícula como un operador epistémico de probabilidad o posibilidad tiene más que ver con el desarrollo de usos similares a los de los aproximantes y atenuantes por parte de este tipo de operadores que con el uso de $\pi$ ov como operador epistémico propiamente dicho.

Los operadores de aproximación en el decir son adverbios y expresiones adverbiales que indican que el hablante considera que un enunciado está expresado en unos términos que no son los más adecuados o exactos ${ }^{9}$. Los operadores de atenuación son elementos similares, pero que el hablante usa para atenuar o mitigar la fuerza de su elocución. Los motivos por los que el hablante busca mitigar su elocución pueden ser variados y han sido sistematizados por Briz y Albelda 2013.

Los elementos de carácter indefinido suelen desarrollar este tipo de funciones a partir de la vaguedad que supone la indefinición. En el caso de los aproximantes, la vaguedad se proyecta sobre la forma en que se emite el enunciado y se reinterpreta como inexactitud. En el caso de los atenuantes, esa vaguedad reduce la fuerza ilocutiva del enunciado, pues el uso de estos elementos supone que los términos en que se expresa no son del todo exactos,

9 Los operadores son marcadores discursivos que solo tienen alcance sobre el segmento que los aloja (Martín-Portolés 1999, p. 4072; Fuentes 2009, pp. 12-13). 
a pesar de que el hablante no los ponga en duda sino para convencer a su interlocutor, autoprotegerse (salvaguardar el yo), reducir el daño a la imagen del otro, evitar desacuerdos y tensiones, crear un ambiente informal, proyectar una imagen cercana o amenizar el discurso.

Una locución adverbial de carácter indefinido que se usa con estas dos funciones en español es «de alguna manera» y sus variantes «de alguna forma» y «de algún modo». Esta locución es un buen término de comparación por haber recibido una atención bastante pormenorizada en la bibliografía y por ser una de las traducciones posibles de $\pi 0 v$, para cuyos valores no literales Montanari (2015, s. u. B) da, por ejemplo, las siguientes posibilidades 'in some way, in some degree, about, probably, perhaps'. Así, Fuentes 2009, s. uu., considera estas locuciones operadores enunciativos cuya función es la de aproximativos en el decir (o aproximantes), esto es, la de indicar que «el término empleado no es el exacto, es una metáfora, un término genérico, o algo cercano o parecido a la intención comunicativa del hablante» (Fuentes 2009, p. 380). Asimismo, reconoce funciones atenuantes cuando el término al que modifican es descortés o fuerte argumentativamente. En este sentido, Llopis 2016 ha hecho un análisis pormenorizado de los usos atenuantes de estas locuciones. De acuerdo con la autora, el valor de indeterminación circunstancial de estas posibilita que asuman con frecuencia un valor de atenuación mucho más abstracto. Sobre un amplio macrocorpus que engloba distintos géneros discursivos, Llopis ha comprobado que se usan como atenuantes, generalmente, como estrategia de autoprotección para salvaguardar el yo eludiendo o minorando responsabilidades a la hora de tratar temas sensibles y, en menor medida, como recurso de prevención de posibles conflictos por la intromisión en el espacio del otro o de una amenaza a la imagen del otro ${ }^{10}$. Véanse los siguientes ejemplos de autoprotección y prevención respectivamente:

Nuestro trabajo pone de manifiesto este problema y esperamos que contribuya de alguna manera a mitigarlo. (La locución permite realizar una afirmación tan tajante con cierto tono de modestia para salvaguardar la propia imagen).

No parece poder negarse que nuestros ordenamientos jurídicos (el alemán tanto como el español) rebosan disposiciones que de alguna manera instru-

10 En este trabajo, no se tiene en cuenta la función no atenuante como retardatario del discurso que Llopis 2016, pp. 114, 117, identifica, por no ser relevante en el caso de $\pi$ ov. 
mentalizan a otro contra su voluntad, en aras de los intereses de otras personas o de la colectividad. (El hablante usa la locución con el objeto de mitigar la crítica que subyace a sus palabras).

Las dos funciones incluyen varios usos (Llopis 2016, p. 114), la de autoprotección: no expresarse de manera categórica, persuadir, cliché o convención retórica, amenizador de la conversación, modestia y evitar riesgos; la de prevención: atenuar críticas y mitigar hechos negativos. Estos usos aparecen clasificados por función en Tabla 1.

Tabla 1. Funciones y usos de los atenuantes.

\begin{tabular}{|c|l|}
\hline Funciones & \multicolumn{1}{c|}{ Usos } \\
\hline & - No expresarse de manera categórica \\
& - Persuadir \\
\hline \multirow{3}{*}{ Autoprotección } & - Cliché o convención retórica \\
& - Amenizador de la conversación \\
& - Modestia \\
& - Evitar riesgos \\
\hline \multirow{2}{*}{ Prevención } & - Atenuar críticas \\
& - Mitigar hechos negativos \\
\hline
\end{tabular}

5. Posición y alcance de $\pi$ ov: de adverbio indefinido de lugar a adverbio de aproximación

Antes de analizar los supuestos usos modales de $\pi$ ov desde la óptica de los operadores de aproximación en el decir y de cortesía, es importante señalar que estos usos son propios del dominio sintáctico-semántico de la claúsula, mientras que la partícula funciona como adverbio de aproximación cuantitativa en el dominio del sintagma.

La partícula $\pi$ ov es un elemento enclítico que ocupa la llamada posición de Wackernagel. Esta posición fue definida, fundamentalmente, por el profesor alemán a finales del s. XIX (Wackernagel 1892). Desde entonces, es muy amplia la lista de publicaciones que se han centrado en la posición de los clíticos, no solo en griego antiguo, sino también en otras lenguas indoeuropeas antiguas y modernas. La última monografía sobre la cuestión en griego antiguo es la de Goldstein 2016, donde el autor defiende que la posición 
de Wackernagel es segunda posición de un dominio sintáctico que puede corresponderse con una oración, una cláusula o un sintagma y que, en el segundo caso, puede ir precedido por elementos extraclausales que no influyen en la segunda posición ${ }^{11}$.

La partícula $\pi$ ov funciona, con su valor local original, en el dominio de la cláusula, ya sea su núcleo un verbo en forma personal, un infinitivo o un participio, pero también en el del sintagma, según su incidencia semántica sea sobre una predicación o sobre un constituyente:

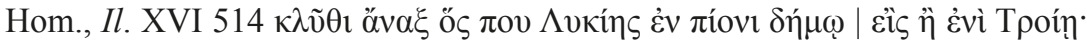
Escucha, señor, que estás en alguna parte de la pingüe tierra de Licia o en

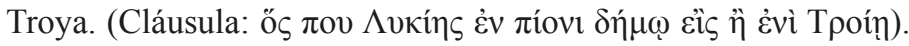

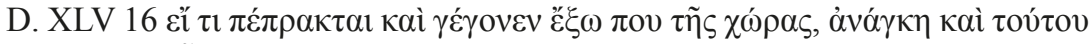

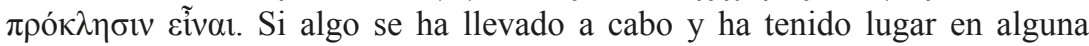
parte fuera del país, es preciso que también haya un requerimiento dirigido a

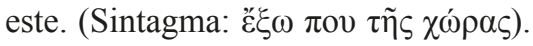

De la misma manera, con los valores no literales, $\pi$ ov puede funcionar en el dominio sintáctico de la cláusula o en el del sintagma. Como adverbio de aproximación cuantitativa funciona en el dominio del sintagma, véase el siguiente ejemplo:

Hdt., VII $22.1[\ldots]$ [

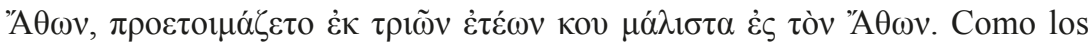
primeros que habían intentado circunnavegar el Atos habían fracasado, se hicieron preparativos durante unos tres años como máximo con respecto al

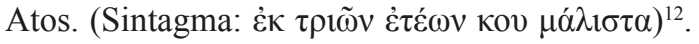

Los usos tradicionalmente considerados modales son propios del dominio de la cláusula. Mi hipótesis es que la partícula significa en los dos casos lo

${ }^{11}$ En concreto, se trata de construcciones de tópico contrastivo y lo que el autor denomina «foco no-monotónico», aquel que corrige o niega alguna de las proposiciones que constituyen la base sobre la que se establece el contexto comunicativo (Goldstein 2016, pp. 176-177).

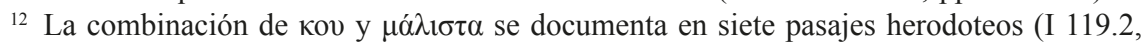
209.2, III 26.3, 120.1, VII 22.1, 223.1, VIII 65.1): mientras que la partícula indica que la cantidad a la que se hace referencia es aproximada, el adverbio señala que dicha cantidad aproximada es el tope máximo que se puede alcanzar. 
mismo, en términos abstractos «aproximación», pero que en el dominio del sintagma funciona como un adverbio de aproximación cuantitativa, mientras que en el dominio de la cláusula funciona como un operador de aproximación en el decir o de atenuación con alcance sobre toda ella. En este sentido, es habitual que los elementos que desarrollan funciones discursivas pasen a funcionar en niveles sintáctico-semánticos superiores (Allan 2017).

\section{6. Пlov modal como operador de aproximación en el decir y de atenuación}

El desarrollo de valores no literales a partir de un significado local es un universal lingüístico. En general, de un significado local se suelen desarrollar, en primera instancia, valores temporales, lo que no es el caso con la partícula griega $\pi$ ov (Koier 2013, pp. 277-279). Por otro lado, el empleo como adverbio epistémico de probabilidad o posibilidad tradicionalmente atribuido a $\pi \mathrm{ov}$ pivotaría sobre el valor indefinido de la partícula: ese valor se reinterpretaría como un valor de aproximación aplicado a la proposición, cuya factualidad pasa a ponerse en cuestión, de donde el sentido epistémico que finalmente expresaría. Véase el siguiente ejemplo, acompañado de una traducción y una glosa que responden a la interpretación tradicional:

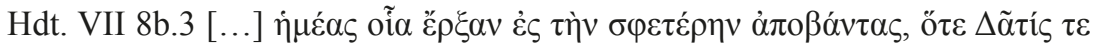

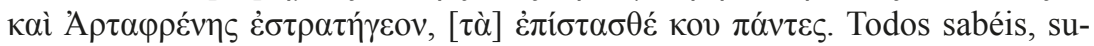
pongo, lo que nos hicieron cuando desembarcamos en su territorio, en la campaña dirigida por Datis y Artáfrenes. (Traducción de Schrader).

De acuerdo con Schrader 1985, p. 34, n. 57 la partícula indica que el propio Jerjes supone que no todos sus soldados tenían conocimiento de esos hechos, pues el Imperio persa era un mosaico de regiones, algunas de ellas muy remotas, y la Primera Guerra Médica no fue más que un acontecimiento de mediana importancia para los persas. Obsérvese que, en general, el supuesto valor epistémico se suele explicar y traducir en términos más propios de los aproximantes y atenuantes (en el pasaje anterior kov se traduce por 'supongo', que es un hedge en la terminología de Caffi 1999 y 2007,

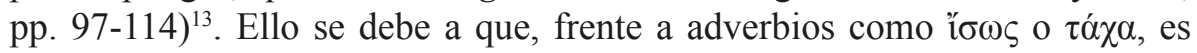

13 Caffi distingue tres tipos de atenuantes: bushes, que matizan el compromiso del hablante con la proposición; hedges, que mitigan su responsabilidad con respecto al acto ilocutivo; 
difícil entender que $\pi$ ov afecte a la vericondicionalidad de su oración, sino que más bien matiza los términos en que se expresa. La consideración de la partícula como un operador epistémico al mismo nivel que los adverbios mencionados se debería a que este tipo de operadores se puede usar también con valores similares con los que el hablante no pone en duda la factualidad de su oración, sino, precisamente, los términos de su expresión (Martí 2008, pp. 79-80; Verano 2016, pp. 139-140). El solapamiento llega al punto de que un operador de modalidad epistémica como $̌ \sigma \omega \varsigma$ desarrolla, asimismo, la

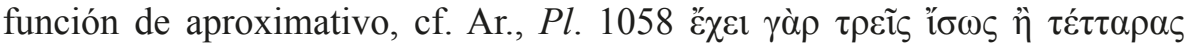
«pues tiene unos tres o cuatro». En este sentido, podemos observar, en griego antiguo, que no solo un adverbio indefinido como $\pi$ ov o adverbios de modalidad epistémica como 'í $\sigma \omega \varsigma$ 'tal vez' desarrollan valores propios de los operadores de aproximación en el decir y de atenuación, también adverbios

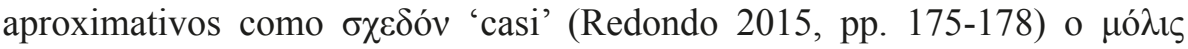
'apenas' (Conti 2017, pp. 11-13). Por lo demás, como hemos visto supra, el desarrollo de estas funciones por parte de $\pi$ ov está ligado a su carácter indefinido, al igual que en el caso de la partícula holandesa ergens 'en alguna parte' (véase n. 6), mientras que en el caso de '̌ $\sigma \omega \varsigma$ tiene que ver con su

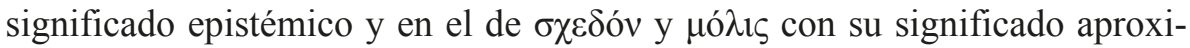
mativo, defectivo o negativo en el caso de $\sigma \chi \varepsilon \delta$ óv y excesivo o positivo en

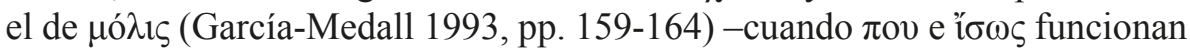
como aproximativos son neutros.

Por su parte, Koier 2013, p. 325 concluye, como ya hemos reseñado, que

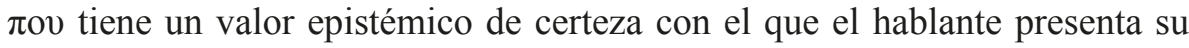
proposición como accesible a su interlocutor, porque se trata de información dada antes, de información que pertenece al conocimiento enciclopédico de ambos o de información que puede inferirse con facilidad a partir del cotexto o el contexto. Koier explica esta evolución, de adverbio local indefinido a adverbio de modalidad epistémica, a partir de una implicatura conversacional en virtud de la cual se infiere del hecho de no ser específico sobre la locali-

\footnotetext{
shields, que adscriben su afirmación a otra fuente o la desplazan a otra situación. Las partículas con significado de vaguedad suelen emplearse como bushes (Llopis 2016, p. 106), aunque las funciones atenuantes de $\pi$ ov son más propias de los hedges, véase Caffi 2007, pp. 102-104. Sobre el uso como atenuante de $\sigma \chi \varepsilon \delta o ́ v$, con el que combina indicar «el grado de compromiso que el hablante tiene con la proposición» con comunicar «contenidos subjetivos que califican el acto verbal concreto que está realizando», véase Redondo 2015, pp. 176-177.
} 
zación que la información que da el hablante es plenamente accesible para su interlocutor. Con todo, advierte que esta evolución se produjo en época prehomérica, por lo que no deja de ser especulativa.

Las conclusiones de Koier, basadas sobre un amplio elenco de traducciones y sobre las propiedades combinatorias de la partícula, ponen de relieve las contradicciones que subyacen a la visión tradicional sobre $\pi$ ov como partícula modal que expresa probabilidad o posibilidad. Sin embargo, no se compadecen con lo que los gramáticos antiguos nos transmitieron sobre dicha partícula ${ }^{14}$. Por otra parte, como la propia autora reconoce, el tratar de establecer los valores de un elemento con un significado tan abstracto como tov a partir de traducciones es un tanto arriesgado y, de hecho, las traducciones suelen equiparar ese elemento con una amplia nómina de adverbios, partículas y expresiones parentéticas de diversos tipos, cf. supra. Mucho menos subjetivo es tratar de establecer sus funciones a partir de sus propiedades combinatorias y, en este punto, llama la atención que $\pi$ ov, adverbio epistémico de certeza, se combine con otros elementos que también aportan certeza, como $\delta \eta \dot{\eta}, \tilde{\eta}$ o $\pi \alpha ́ v \tau \omega \varsigma$. En este sentido, las descripciones tradicionales consideran que $\pi$ ov aporta a esas combinaciones una atenuación de la certeza que el otro elemento expresa, véase Denniston 1954, pp. 285-286, 267-268, sobre

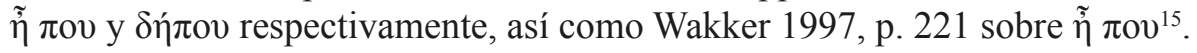
Por último, el uso de $\pi$ ov asociado con proposiciones cuya certeza no se discute, aunque posible, resulta tautológico, sobre todo, cuando es tan frecuente como en los diálogos platónicos. También es relevante su frecuencia

${ }^{14}$ Es difícil interpretar los escasos comentarios de los gramáticos antiguos sobre $\pi$ ov que nos han llegado. Por un lado, el carácter expletivo que le atribuye Apolonio Díscolo y, de forma menos evidente, la glosa de la Suda o la entrada del Etymologicum Gudianum es poco aclaratorio. Por otro lado, la identificación de $\pi$ ov con ı̌ $\sigma \omega \varsigma$, $\sigma \chi \varepsilon \delta \delta$ (en $\tilde{\eta} \pi \mathrm{ov}$ ) y con $\pi$ o $\lambda \lambda \tilde{\omega}$

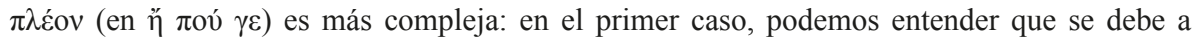
que los adverbios î́ $\sigma \varsigma$ y $\sigma \chi \varepsilon \delta o ́ v$ también podían usarse como atenuantes, mientras que en el segundo es posible que la identificación tenga que ver con la adición de un segundo miembro a la disyunción.

${ }^{15}$ Un estudio específico de $\tilde{\eta}$ Tov puede encontrarse en Zakowski 2014, donde el autor propone traducir esta secuencia por 'surely, no doubt'. Este trabajo se enmarca dentro de la teoría de la relevancia de Sperber y Wilson, y Zakowski concluye que «ĩ $\pi$ ov can mark utterances which are assumptions about what the speaker thinks the hearer has inferred, or assumptions about what the speaker thinks the hearer should infer». En una línea interpretativa similar, véase Bonifazi 2016, IV.4 §112. 
con formas de la primera persona de verbos de decir y saber, pues es frecuente, en las lenguas del mundo, que el hablante mitigue la fuerza asertiva de sus palabras o pensamientos (Conti 2017, p. 11).

En mi opinión, los usos no literales de $\pi$ ov giran todos en torno al valor etimológico de indefinición de la partícula (véase supra), pero tienen una explicación mucho más directa que la tradicional o la de Koier. Con este valor la partícula desarrollaría, en una evolución ampliamente documentada en otras lenguas ${ }^{16}$, la función de adverbio de aproximación en el decir ${ }^{17}$. Este tipo de marcadores se usan cuando el hablante no se compromete del todo o no está satisfecho con la forma en que ha emitido su enunciado:

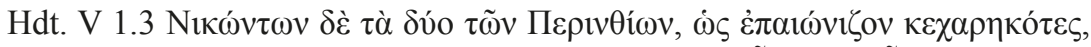

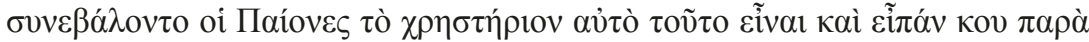

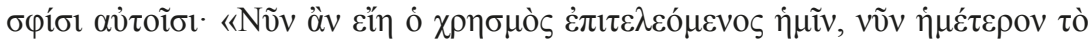
ह̌ $p \gamma o v »$. Al vencer los perintios en dos (de los duelos), mientras lo celebraban entonando el peán, los peonios comprendieron que el oráculo era esto mismo y, poco más o menos, se dijeron a sí mismos: «Ahora puede que se haya cumplido la profecía para nosotros, ahora nos toca actuar».

En este caso Heródoto reproduce las palabras que se dirían los peonios a sí mismos, pero usa la partícula para indicar que las palabras exactas no tienen por qué coincidir con las que él les atribuye.

El desarrollo de un valor de aproximación es un desarrollo de tipo metafórico que pivota sobre el carácter indefinido de $\pi$ ov, que originalmente indica posición en un lugar indeterminado. Ese mismo carácter es el que motiva su uso como refuerzo de otros elementos indefinidos (véase supra), refuerzo que también se documenta en el caso de hol. ergens. En general, la evolución de un adverbio local a marcador del discurso se puede considerar un

${ }^{16}$ Llopis 2016, p. 107 distingue la categoría de la «indeterminación de las circunstancias de lo predicado» como una de las categorías en las que pueden clasificarse los «aproximadores» que Prince et al. 1982 denominan «adaptadores», los que afectan al contenido mediante la adaptación de una expresión a una situación que no es prototípica. Los adverbios que estamos estudiando se insertarían dentro de esa categoría.

${ }_{17}$ Verano 2016, pp. 133-135 atribuye a la partícula la categoría de «comentario metadiscursivo», con el que el hablante toma cierta distancia de los términos en que realiza su formulación. De acuerdo con su exposición, este uso se desarrolla a partir del carácter indefinido de $\pi$ ov, pero estaría relacionado con el valor epistémico generalmente atribuido a esta partícula. 
caso de subjetificación, en virtud de la cual un término lingüístico se dota de significados de tipo metatextual relacionados con la actitud o la evaluación del hablante, cf. Traugott 2010.

Esta evolución ha debido de producirse a partir de contextos puente como los siguientes:

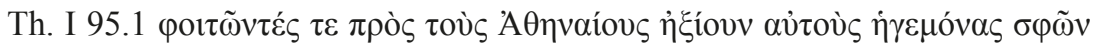

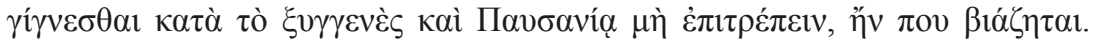
Acudiendo en repetidas ocasiones a los atenienses, demandaron que se erigieran en sus caudillos, en razón de su origen común, y que no cedieran ante Pausanias si cometía alguna violencia.

En este pasaje, los jonios solicitan a los atenienses que sustituyan al espartano Pausanias en el mando de las tropas griegas que reconquistaron $\mathrm{Bi}$ zancio a los persas por la actitud despótica de este. En la oración condicional

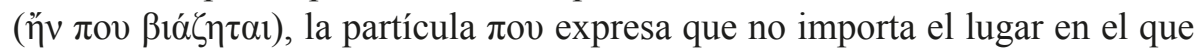
cometa violencia; si lo hace, Pausanias debe ser destituido. El contexto es tan impreciso que la lectura de $\pi$ ov queda abierta a otro tipo de interpretaciones no locales, cf. Koier 2013, pp. 179-187.

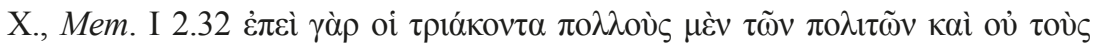

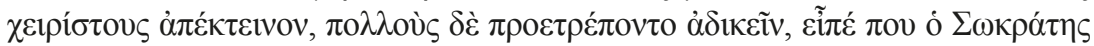

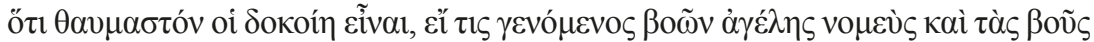

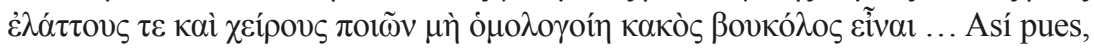
cuando los Treinta condenaron a muerte a muchos ciudadanos, y no los peores, e impelieron a otros muchos a cometer injusticia, dijo Sócrates que le parecería inaudito si alguien que fuera pastor de un rebaño de vacas e hiciera que las vacas fueran menos y peores no estuviera de acuerdo en que es un mal boyero.

Es relativamente frecuente el uso de esta partícula cuando se citan las

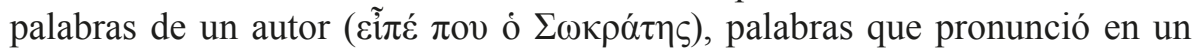
lugar indeterminado o que se sitúan en un lugar indeterminado de su obra (Koier 2013, pp. 289-290). En este contexto, se puede entender también que las palabras que se citan son aproximadas, como aproximado es el lugar en el que se pronunciaron o en el que aparecen recogidas ${ }^{18}$.

${ }_{18}$ Estos contextos puente sugieren que la proyección metafórica se basa en inferencias invitadas con una base metonímica. Se trataría de un caso de metaphtonymy, es decir, de 
A partir de este valor de aproximación en el decir, la partícula se usa, asimismo, cuando el hablante no pone en cuestión la adecuación o exactitud de los términos en que se expresa, sino que mitiga su fuerza argumentativa. Se trata de una implicatura del tipo de las incluidas por Traugott-Dasher 2001, pp. 34-35 en su «Invited Inferencing Theory of Semantic Change». Estas inferencias invitadas tienen una base metonímica en virtud de la cual una expresión desarrolla significados contextuales relacionados con su sentido original que pueden generalizarse a otros contextos y, finalmente, reanalizarse como significados semánticos de pleno derecho. Por lo demás, el desarrollo de significados intersubjetivos a partir de significados subjetivos es habitual en este tipo de elementos, esto es, el desarrollo de sentidos relacionados con la actitud del hablante con respecto a su interlocutor a partir de sentidos relacionados con la actitud o evaluación del hablante ${ }^{19}$. De hecho, son estos usos de carácter intersubjetivo los que explicarían su asociación con proposiciones cuya certeza está fuera de duda o con otros operadores que el hablante utiliza cuando quiere subrayar la certeza de la proposición ${ }^{20}$. La evolución semántica de la partícula $\pi$ ov aparece recogida esquemáticamente en Gráfico 1.

La partícula $\pi$ ov con los valores tradicionalmente considerados modales es muy frecuente en los poemas homéricos y en los diálogos platónicos, así como en el drama y en los pasajes dialogados de Heródoto, pero, por ejemplo, está prácticamente ausente de los discursos de los oradores ${ }^{21}$, véase Tabla 2 , donde se recoge el número de ejemplos de $\pi$ ov clasificados por tipo de uso (local,

interacción de metáfora y metonimia (Goossens 1990; Ruiz-Díez 2002, pp. 518-527), más concretamente, de una metáfora de metonimia. El uso de $\pi$ ov como adverbio de aproximación en el sintagma responde a una evolución similar (metonimia dentro de metáfora): el adverbio indica lugar indeterminado en la serie invocada por la cifra a la que modifica, de donde su uso como adverbio de aproximación. En este caso la proyección es del dominio de lo local al de la cantidad, mientras que en el anterior es del dominio de lo local al de la manera.

${ }^{19}$ El término subjetificación es un término general que engloba el más concreto de intersubjetificación, este último aplicado específicamente a los significados que surgen de la actitud del hablante con respecto a su interlocutor. Cf. Traugott 2010.

${ }^{20}$ Véase cómo todos los sentidos de $\pi$ ov se mantienen sincrónicamente, fenómeno denominado layering (Hopper-Traugott 2003, pp. 124-126).

${ }^{21}$ Cf. Denniston 1954, pp. 490-495, y Sicking 1993, pp. 57-59; sobre las frecuencias de

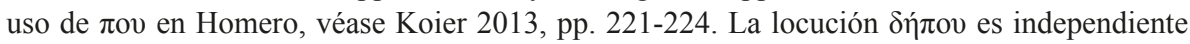
y, en nuestro análisis, tampoco tenemos en cuenta $\tilde{\eta} \pi$ ov. 
Gráfico 1. Evolución semántica de $\pi$ ov. SENTIDO OBJETIVO

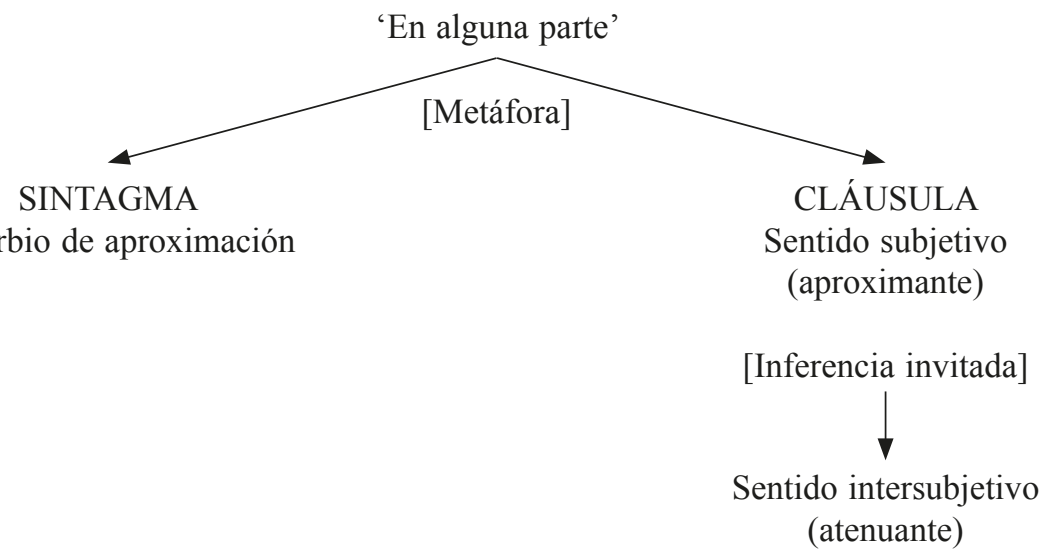

Tabla 2. Distribución de $\pi$ ov por usos y autores.

\begin{tabular}{|l|c|c|c|c|c|}
\cline { 2 - 6 } \multicolumn{1}{c|}{} & Local & Indefinido & Aproximativo & Aproximante & Atenuante \\
\hline Heródoto & 4 & 11 & 12 & 1 & $18^{22}$ \\
\hline Sófocles & 12 & 6 & - & - & 24 \\
\hline Aristófanes & 14 & 13 & - & 1 & 22 \\
\hline Platón & 24 & 23 & 2 & 16 & 181 \\
\hline Demóstenes & 19 & 2 & - & 3 & 1 \\
\hline
\end{tabular}

reforzando otras expresiones indefinidas, aproximativo, aproximante y atenuante) en las obras de Heródoto, Sófocles, Aristófanes, Platón (Eutifrón, Apología, Critón, Fedón, Cratilo, Teeteto, Sofista y Político) y Demosténes.

En este sentido, su distribución no se compadece del todo con los géneros en los que se ha observado que suelen usarse los denominados atenuantes en español (Briz y Albelda 2013; Llopis 2016) ${ }^{23}$. En general, los atenuantes son

${ }^{22}$ De estos 18 ejemplos, 7 están en pasajes dialogados y 3 en oráculos.

${ }^{23}$ Sobre 'por así decir' y sus variantes, véase Fuentes 2009 y DPDE s. uu. 
propios de contextos formales y más abundantes en la prosa escrita que en el discurso oral. No obstante, Llopis ha documentado el uso de «de alguna manera / forma / modo» tanto en géneros formales como en la conversación informal; además, encuentra que la locución «por decirlo de alguna manera» y sus variantes, que también funcionan como aproximantes y como atenuantes, se emplean más en entrevistas que en géneros formales. Por lo demás, más allá de que el uso de la partícula griega sea más propio de textos dialógicos que de textos monológicos, se trata de textos literarios muy estilizados $^{24}$. Sea como fuere, buena parte de los usos de $\pi$ ov coinciden con los usos establecidos para los atenuantes por estos autores. Véanse los siguientes ejemplos, todos ellos incluidos en Tabla $2^{25}$ :

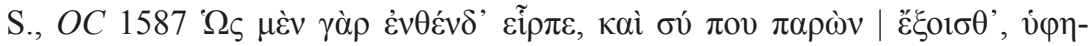

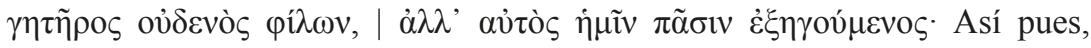
cómo se marchó de aquí también tú, que estabas presente, sabes de alguna manera, sin que lo guiara amigo alguno, sino guiándonos él a todos nosotros. (Uso: no expresarse de manera categórica)

En la parte final de la tragedia de Edipo en Colono un mensajero vuelve a donde está el coro para anunciarle que Edipo ha muerto. El coro pregunta cómo ha sucedido. El mensajero responde que ellos mismos lo han visto marcharse de donde están para morir, afirmación que viene mitigada por el uso de la partícula, pues puede parecer no solo sorprendente, sino también chocante. Se debe subrayar que no cabe interpretar $\pi$ ov con sentido local modificando al participio $\pi \alpha \rho \omega ́ v$, primero por su posición (se esperaría más bien $\pi \alpha \rho \omega ́ v \pi o v$ ), luego porque el contexto deja perfectamente claro dónde estaba presente el coro. Por lo demás, se trata de un ejemplo de la asociación de $\pi$ ov con un verbo de conocimiento ( $\check{\xi} \xi o 1 \sigma \theta \alpha$ ), cf. Koier 2013, pp. 258-259.

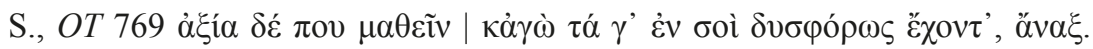
Creo que soy digna, también yo, de saber lo que te que te angustia, Señor. (Uso: persuadir)

${ }^{24}$ En el caso de los diálogos platónicos, Shalev (2017) atribute el uso de $\pi$ ov y otros atenuantes a la urbanitas ática.

${ }_{25}$ Únicamente no he encontrado ejemplos de la partícula usada para expresar modestia, uso que Llopis 2016, p. 116 describe como propio de artículos académicos y con el que se busca expresar su propósito de manera modesta. 
En este verso, Yocasta le pide a Edipo que comparta con ella los temores que alberga después de que esta le contara que, muerto Layo y tras aparecer el propio Edipo en Tebas, el único superviviente del asesinato del primero le suplicó que lo enviara al monte como pastor. El uso de la partícula hace que el tono de la petición de Yocasta sea más sugerente. Véase que, en general, Yocasta se dirige a Edipo con respeto, tratándolo de ớv $\alpha \xi$ 'señor, soberano'.

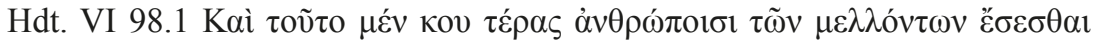

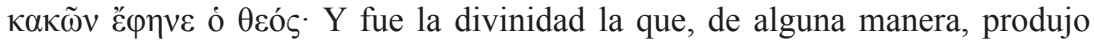
este hecho como una señal para los hombres de los males que iban a venir. (Uso: convención retórica)

Koier señala la frecuente asociación de esta partícula con oraciones en las que se atribuye una acción a la divinidad. Podemos englobar estos casos en los que Llopis 2016, p. 115 califica como usos en los que este tipo de elementos sirven como cliché o convención retórica para hacer el lenguaje menos rígido.

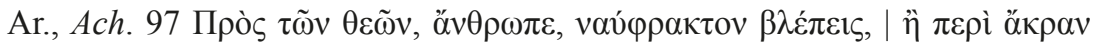

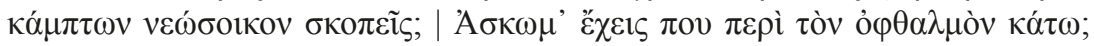
Por los dioses, hombre, tienes mirada de barco de guerra, ¿o es que buscas fondeadero después de doblar el cabo? En cierto modo, tienes una badana para remos alrededor del ojo. (Uso: amenizador)

En este ejemplo, el uso de la partícula también tiene por objeto hacer el lenguaje menos rígido, pero, a su vez, es de carácter puramente interactivo para crear una atmósfera distendida que en el caso de la comedia aristofánica no está reñida con la mofa: Aristófanes compara los ojos de la máscara del personaje con los ojos por donde salían los remos de las trirremes.

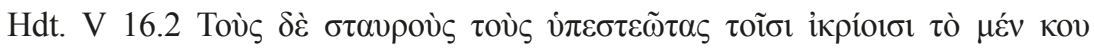

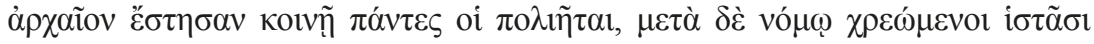

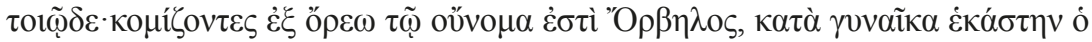

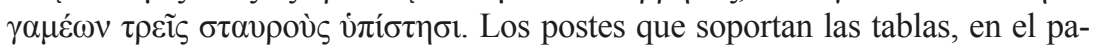
sado, de alguna manera los colocaron los habitantes en común, pero después los colocan sirviéndose de la siguiente costumbre: los traen de un monte llamado Orbelo y cada vez que cada uno se casa coloca tres postes. (Uso: evitar riesgos)

La estrategia de autoprotección se basa en adelantarse a las críticas que puedan recibirse de parte del interlocutor. En este caso, Heródoto describe el 
sistema de hábitat palafítico de los peonios del lago Prasíade: cuando Heródoto escribe, la plataforma sobre la que residen se apoya sobre postes que los hombres colocan cada vez que se casan, pero en el pasado, Heródoto hipotetiza que los postes debieron de haber sido colocados de forma solidaria, hipótesis que podría ser criticada o refutada.

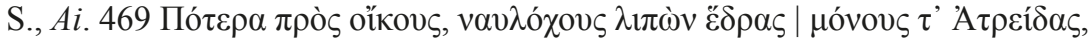

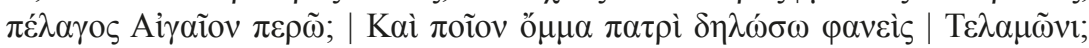

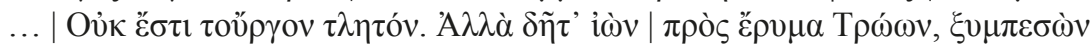

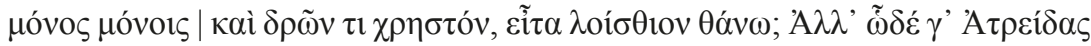

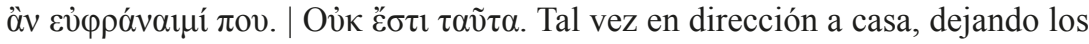
lugares que nos sirven como puerto y solos a los Atridas, cruce el mar Egeo. ¿Y con qué cara me presentaré ante mi padre Telamón? ... No es una carga soportable. O tal vez me dirija contra la fortificación de los troyanos y, tras batirme solo contra ellos uno por uno y hacer algo útil, muera luego por fin. Pero así podría agradar, en cierto modo, a los Atridas. Eso no es una opción. (Uso: atenuar críticas)

En este caso, Áyax se pregunta cuál debe ser su reacción ante la ofensa que se le ha infligido al otorgar los jefes aqueos, Agamenón y Menelao, la armadura del fallecido Aquiles a Odiseo en lugar de a él mismo. Una opción es seguir combatiendo contra los troyanos, lo que supondría agradar a los

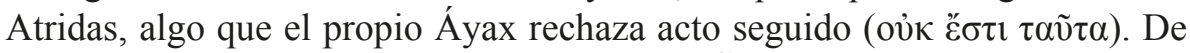

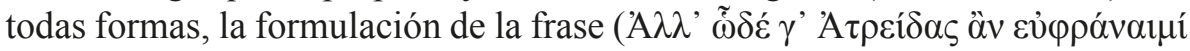
$\pi \mathrm{ov})$ incluye la partícula con el objeto de atenuar las críticas que él mismo se dirige en un monólogo en el que Áyax se desdobla para evaluar los pros y los contras de sus opciones.

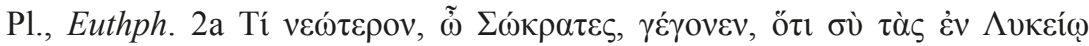

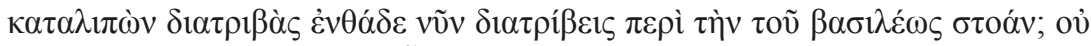

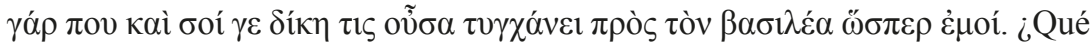
hay de nuevo, Sócrates, que has abandonado tus ocupaciones en el Liceo y ahora te entretienes aquí junto al Pórtico del Rey? No creo que también te encuentres en la tesitura de tener un pleito de los del arconte rey, como me sucede a mí. (Uso: mitigar hechos negativos)

En este ejemplo, que abre la obra, Eutifrón pregunta a Sócrates el motivo que le trae al Pórtico del Rey, pues allí el arconte rey se encargaba de las 
acusaciones de homicidio e impiedad. La partícula se usa para mitigar las implicaciones negativas de que Sócrates se encuentre en dicho lugar.

\section{Conclusiones}

La partícula $\pi$ ov tiene un significado original «en alguna parte» de tipo local e indefinido, pero ya desde los primeros textos literarios se emplea con valores no literales que se han desarrollado a partir del local. Tradicionalmente, se ha considerado que buena parte de estos valores era de carácter epistémico y que, con ellos, la partícula expresa probabilidad o posibilidad. Recientemente, Koier ha rebatido la interpretación tradicional para llegar a la conclusión de que la partícula expresa, más bien, certeza. En este trabajo hemos visto cómo los valores modales de $\pi$ ov se pueden entender mejor desde la óptica de los operadores de aproximación en el decir y de atenuación: la partícula habría desarrollado, metafóricamente, usos como aproximante a partir del valor indefinido original por un proceso de subjetificación, usos con los que se emplearía, asimismo, como atenuante por inferencia pragmática en una evolución propia de este tipo de elementos (subjetivo > intersubjetivo). Esos valores se compadecen con su uso como adverbio de aproximación cuantitativa o aproximativo, otro uso no literal derivado de su significado indefinido y que es propio del dominio del sintagma, mientras que las funciones discursivas de aproximante y atenuante son propias del dominio de la cláusula. Frente a otros adverbios que desarrollan funciones discursivas similares, como el epistémico ǐ $\sigma \omega \varsigma$ o los aproximativos $\sigma \chi \varepsilon \delta \delta$ y y $\mu$ ó $\lambda$ is, la evolución de $\pi$ ov es comparable con la de hol. ergens, otro adverbio local de carácter indefinido que también presenta funciones (inter)subjetivas derivadas de ese valor indefinido.

\section{BiBLIOGRAFÍA}

Allan, R. 2017: «The grammaticalization of Greek particles», en Logozzo, F. y Poccetti, P. (eds.), Ancient Greek Linguistics. New Approaches, Insights, Perspectives, Berlín - Boston, pp. 103-118.

Bailly, A. 20004: Dictionnaire grec-français, rédigé avec le concurs de E. Egger, París. Bodin, L. y Mazon, P. 1912: Extraits d'Aristophane et Ménandre, París.

Bolling, G. M. 1929: «The meaning of $\pi$ ov in Homer», Language 5/2, pp. 100-105. Bonifazi, A., Drummen, A. y de Kreij, M. 2016: Particles in Ancient Greek discourse. Five volumes exploring particle use across genres, Washington DC, [https:// chs.harvard.edu/CHS/article/display/6391]. 
Briz, A. y Albelda, M. 2013: «Una propuesta teórica y metodológica para el análisis de la atenuación lingüística en español y portugués. La base de un proyecto común (ES.POR.ATENUACIÓN)», Onomázein 28, pp. 288-319.

Caffi, C. 1999: «On mitigation», Journal of Pragmatics 31, pp. 881-909.

Caffi, C. 2007: Mitigation, Ámsterdam.

Conti Jiménez, L. 2017: «Sobre la expresión del esfuerzo y de la aproximación: análisis de $\mu o ́ \gamma ı \varsigma$ y $\mu$ ó $\lambda ı \varsigma$ en griego antiguo», Emerita 85 (1), pp. 1-25.

Denniston, J. D. 1954²: Greek particles, Oxford.

$D P D E=$ Briz, A., Pons, S. y Portolés, J. (coords.) 2008: Diccionario de partículas discursivas del español, < http://www.dpde.es > (12/02/2018).

Fuentes, C. 2009: Diccionario de conectores y operadores del español, Madrid.

García-Medall Villanueva, J. A. 1993: «Sobre casi y otros aproximativos», Dicenda. Cuadernos de Filología Hispánica 11, pp. 153-170.

Goldstein, D. 2016: Classical Greek Syntax. Wackernagel's Law in Herodotus, Leiden-Boston.

Goossens, L. 1990: «Metaphtonymy: the interaction of metaphor and metonymy in expressions for linguistic action», Cognitive Linguistics 1/3, pp. 323-340.

Hopper, P. J. y Traugott, E. 2003²: Grammaticalization, Cambridge.

Huitink, L. 2009: «Pragmatic Presupposition and Complementation in Ancient Greek», en Bakker, S. J. y Wakker, G. C. (eds.), Discourse Cohesion in Ancient Greek, Leiden-Boston, pp. 21-40.

Italie, G. 1955: Index Aeschyleus, Leiden.

Kiparsky, P. y Kiparsky, C. 1970: «Fact», en Bierwisch, M. y Heidolph, K. E (eds.), Progress in Linguistics, La Haya, pp. 143-173.

Koier, E. 2013, Interpreting particles in dead and living languages: a construction grammar approach to the semantics of Dutch ergens and Ancient Greek pou, Tesis doctoral de la Universidad de Leiden.

Liddell, H. G., Scott, R. y Jones, H. S. 19969: A Greek-English lexicon. With a revised supplement, Oxford.

Llopis Cardona, A. 2016: «De la indeterminación a la atenuación: de alguna maneral forma/modo», RILI 14, pp. 105-121.

Martí Sánchez, M. 2008: Los marcadores en español L/E: conectores discursivos y operadores pragmáticos, Madrid.

Martín Zorraquino, M. A. y Portolés Lázaro, J. 1999: «Los marcadores del discurso», en Bosque, I. y Demonte, V. (eds.), Gramática descriptiva de la lengua española, Madrid, pp. 4051-4213.

Montanari, F. 2015: The Brill Dictionary of Ancient Greek, Boston.

Prince, E. F., Frader, J. y Bosk, C. 1982: «Hedgings in physician-physician discourse», en Di Pietro, R. J. (ed.), Linguistics and the professions, Norwood, pp. 83-97. 
Redondo Moyano, E. 2015: «Adverbios de foco y marcadores discursivos: $\sigma \chi \varepsilon \delta o ́ v$ en la novela griega antigua», Minerva 28, pp. 163-183.

Rijksbaron, A. 20063: The Syntax and Semantics of the Verb in Classical Greek. An Introduction, Chicago - Londres.

Ruiz de Mendoza, F. J. y Díez, O. 2002: «Patterns of Conceptual Interaction», en Dirven, R. y Pörings, R. (eds.), Metaphor and Metonymy in Comparison and Contrast, Berlín - Nueva York, pp. 489-532.

Schrader, C. 1977-1989: Heródoto, Historia, cinco volúmenes, Madrid.

Schwyzer, E. y Debrunner, A. 1950: Griechische Grammatik. Zweiter Band: Syntax und syntaktische Stilistik, Múnich.

Scolnicov, S. 2003: Plato's Parmenides, Berkeley.

Shalev, D. 2017: «Attenuated, modified, assent-seeking declaratives, interrogation and urbanitas in the Greek of Platonic dialogue», en Logozzo, F. y Poccetti, P. (eds.), Ancient Greek Linguistics. New Approaches, Insights, Perspectives», Berlín - Boston, pp. 429-446.

Sicking, C. M. J. 1993: «Appendix: 3. đov», en Sicking, C. M. J. y van Ophuijsen, J. M., Two studies in Attic particle usage: Lysias and Plato, Leiden - Nueva York - Colonia, pp. 57-64.

Slater, W. J. 1969: Lexicon to Pindar, Berlín.

Traugott, E. C. 2010: «(Inter)subjectivity and (Inter)subjectification: A reassessment», en Davidse, K., Vandelanotte, L. y Cuyckens, H. (eds.), Subjectification, Intersubjectification and Grammaticalization, Berlín-Nueva York, pp. 29-74.

Traugott, E. C. y Dasher, R. B. 2001: Regularity in Semantic Change, Cambridge.

Verano Liaño, R. 2016: «El comentario metadiscursivo en griego antiguo: aproximación desde la lengua de Platón», RSEL 46/1, pp. 123-142.

Wackernagel, J. 1892: «Über ein Gesetz der indogermanischen Wortstellung», IF 1, pp. 333-436.

Wackernagel, J. 1895: «Miszellen zur griechischen grammatik 24», KZ 33, pp. 21-24.

Wakker, G. 1994: Conditions and Conditionals: An Investigation of Ancient Greek, Ámsterdam.

Wakker, G. 1997: «Emphasis and Affirmation. Some Aspects of $\mu$ ท́ $v$ in Tragedy», en Rijksbaron, A. (ed.), New Approaches to Greek Particles, Ámsterdam, pp. 209-231.

Zakowski, S. 2014: «Inference and metarepresentation: Ancient Greek $\tilde{\tilde{\eta}} \pi$ ov as a constraint on high-level explicatures», Journal of Pragmatics 74, pp. 109-128.

Fecha de recepción de la primera versión del artículo: 14/02/2018

Fecha de aceptación: 29/04/2018

Fecha de recepción de la versión definitiva: 05/09/2018 